\title{
ILC BEAM ENERGY MEASUREMENT BASED ON SYNCHROTRON RADIATION FROM A MAGNETIC SPECTROMETER
}

\author{
K. Hiller, H.J. Schreiber, DESY, Zeuthen, Germany \\ R. Makarov, Moscow State University, Moscow, Russia \\ E. Syresin, B. Zalikhanov, Joint Institute for Nuclear Research, Dubna, Russia
}

\begin{abstract}
A magnetic spectrometer with a relative energy resolution of $5 \cdot 10^{-5}$ was proposed for ILC beam energy calibration. A complementary method of beam energy measurement based on registration of synchrotron radiation (SR) from the dipole magnets of the energy spectrometer is proposed. Measuring both horizontal edge positions of the SR fan at a distance of 50-70 m downstream of spectrometer allows to determine the beam energy with high accuracy. The main principals of the SR beam energy measurement, first numerical simulations by means of GEANT and physical limitations of the detector coordinate determination are described. Two versions of novel detectors for low-energy $(<20 \mathrm{keV})$ photon registration are proposed. They are characterized by a high counting rate and a linear resolution of $2-3 \mu \mathrm{m}$.
\end{abstract}

\section{INTRODUCTION}

A magnetic spectrometer with an energy resolution of $\Delta E / E=5 \cdot 10^{-5}$ was proposed for ILC beam energy calibration [1]. The measurement is based on precise determination of beam positions with a resolution of 100 $\mathrm{nm}$ and the spectrometer B-field integral with a relative accuracy of $2 \cdot 10^{-5}$. The electrons/positrons which pass through the spectrometer magnets produce synchrotron radiation. Complementary, the beam energy is proposed to be measurable with an uncertainty of $\Delta E / E \cong 5 \cdot 10^{-5}$ by monitoring synchrotron radiation photons within the energy range of $1-20 \mathrm{keV}$ and a high-spatial resolution detector allowing coordinate measurements with 2-3 $\mu \mathrm{m}$ precision.

An energy spectrometers based on SR was used at SLAC for precision measurements of the SLC beam energy [23]. The SLAC SR spatial detectors measured the beam position with an accuracy of $30 \mu \mathrm{m}$ and the $45 \mathrm{GeV}$ beam energy was determined with a resolution better than 250 ppm [2-3].

\section{SYNCHROTRON RADIATION FROM THE ENERGY SPECTROMETER}

The ILC spectrometer proposed in [1] consists of two ancillary magnets and one spectrometer magnet (see Fig.
1). The spectrometer magnetic rigidity corresponds to $0.84 \mathrm{~T} \cdot \mathrm{m}$ and the nominal beam energy is $250 \mathrm{GeV}$. The length of spectrometer magnet is $3 \mathrm{~m}$, the distance between the ancillary and spectrometer magnets is $10 \mathrm{~m}$ and the length of the ancillary magnet $1.5 \mathrm{~m}$.

We assume that the SR detector is placed $50 \mathrm{~m}$ downstream of the spectrometer magnet. The horizontal spot size and the positions of both edges of the SR fan are defined by the deflection angles in the magnets, the beam energy and the geometry of the set-up. The deflection angle in the bending plane $\theta / 2=e B l / 2 \gamma m c$ depends on the magnetic rigidity $B l$ and the beam energy. It results to $\theta$ $\approx 1$ mrad for a beam energy of $250 \mathrm{GeV}$ and a magnetic rigidity of $0.84 \mathrm{~T} \cdot \mathrm{m}$ respectively a magnetic field strength of $0.28 \mathrm{~T}$.

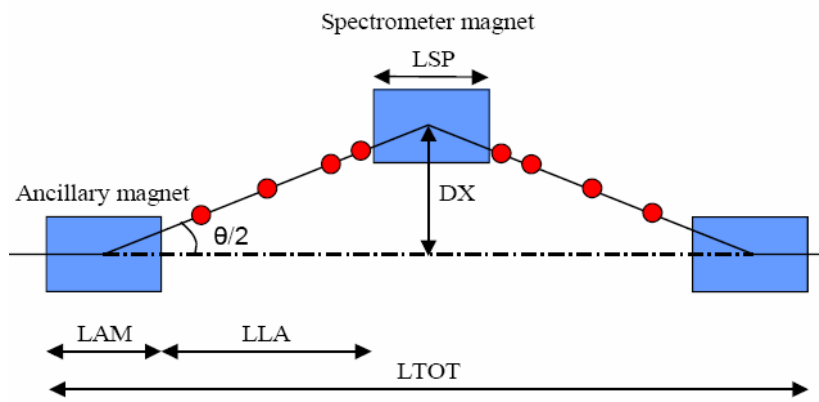

Figure 1: Scheme of magnetic spectrometer proposed for ILC beam energy measurements [1].

The position of the left horizontal SR spot edge $x_{R}=L_{l} \theta / 2$ $\approx 3.1 \mathrm{~cm}$ is defined by the distance between the first ancillary magnet and the detector, $\mathrm{L}_{1}=61.5 \mathrm{~m}$, and the deflection angle. The position of the right horizontal edge $x_{L}=L_{2} \theta / 2 \approx 2.4 \mathrm{~cm}$ is proportional to the distance between the center of the spectrometer magnet and the detector, $\mathrm{L}_{2}=48.5 \mathrm{~m}$. Hence, the horizontal spot size corresponds to $x=x_{L}+x_{R} \approx 5.5 \mathrm{~cm}$. The $\mathrm{SR}$ fan horizontal size is inversely proportional to the electron energy $x \propto \gamma^{-1}$. This variation constitutes the basic concept of monitoring the beam energy.

The vertical SR spot size depends on the photon energy. For photons with an energy close to the critical energy of $\varepsilon_{\mathrm{cr}} \approx 11.4 \mathrm{MeV}, \mathrm{E}=250 \mathrm{GeV}$ and $\mathrm{B}=0.28 \mathrm{~T}$ the divergence angle is equal to $\theta_{\mathrm{SR}} \approx \gamma^{-1} \approx 2 \mu \mathrm{rad}$, while the divergence angle for soft $20 \mathrm{keV}$ photons equals to $\theta_{\mathrm{sr}} \approx 12 \mu \mathrm{rad}$. The 
vertical spot size for such $\gamma$-quanta corresponds to $y \approx \mathrm{L}_{1} \theta_{\text {sr }}$ $\approx 0.6 \mathrm{~mm}$.

The $1 / \gamma$-variation of the horizontal SR fan edge position permits to measure the beam energy with a resolution of $\Delta E / E \approx\left[(\Delta x / x)^{2}+(\Delta B l / B l)^{2}+(\Delta L / L)^{2}\right]^{1 / 2} \approx 5 \cdot 10^{-5}$ for a detector resolution of $\Delta x \approx 2 \mu \mathrm{m}$ (or a relative detector uncertainty of $\Delta x / x \approx 5 \cdot 10^{-5}$ ), a relative accuracy of the magnetic rigidity of $\triangle B l / B l \approx 2 \cdot 10^{-5}$ and an error for the distance to the detector of $\Delta L / L \approx 5 \cdot 10^{-6}$. The last two requirements seem to be feasible, while the most critical quantity is $\Delta x / x$.

One of the sources for SR spot size spreading is related to the electron energy spread within a bunch. The electron energy spread, expected to be of $\Delta \mathrm{E} / \mathrm{E} \approx 10^{-3}$, leads to a spreading of the SR horizontal spot size of $x^{E}{ }_{\text {edge }}=L_{l} \theta / 2 \cdot \Delta E / E \approx 25 \mu \mathrm{m}$ in the detector.

The fringe fields of the magnets produce additional SR and increase the horizontal fan size. Although the integrated fringe field for each magnet only constitutes a very small fraction of the total B-field integral, its impact on the SR yield close to the edges is non-negligible and has to be accounted for in detailed studies.

Another source of edge position dilution is related to the photon angular spread. It is expected to be of the order of $\Delta x^{S R} \approx L_{1} \theta_{s r} \approx 600 \mu \mathrm{m}$.

Interactions of hard SR photons with the vacuum pipe material are an important source for additional $\gamma$-quanta with low energy and increase the SR fan horizontal size. Avoiding such interactions is an essential constraint for the design of the set-up.

As a result, the sensitive horizontal width of the detector area has to be in the range of $3 \mathrm{~mm}$ for registration of soft SR quanta. This area has furthermore to accommodate possible jitters of the beam position, angle and charge as well as to provide a sufficient large $x$-range for SR endpoint fitting procedures.

In summary, two SR detectors with a sensitive region of about $x_{d e t} \approx 3 \mathrm{~mm}$ and high-spatial resolution are required to measure the horizontal edge positions of the fan which covers a range of some $5 \mathrm{~cm}$.

\section{EXPERIMENTAL CONDITIONS}

The approximate number of $\gamma$-quanta produced in the SR fan will be $N_{0} \approx 2 \cdot 10^{11}$ for a bunch intensity of the $2 \cdot 10^{10}$, an electron energy of $250 \mathrm{GeV}$ and the dipole fields as specified in [1]. The number of photons with energies of $\Delta \epsilon \approx 1-20 \mathrm{keV}$ entering an active detector area of $x_{d e t}=1$ $\mathrm{mm}$ is estimated as $N_{\gamma} \approx N_{0}\left(\Delta \varepsilon / \varepsilon_{c r}\right)^{1 / 3} \cdot x_{\text {det }} / x \approx 5 \cdot 10^{8}$, where $\epsilon_{c r}$ is the critical photon energy and $x$ is the horizontal SR spot size. From the comparison of $N_{\gamma}$ with $N_{0}$ it follows that the background SR component is much greater than the number of useful soft $\gamma$-quanta. Therefore, a highly selective reflecting mirror should be used to separate the interesting $\gamma$-quanta with energies below $20 \mathrm{keV}$

For mirrors with a large atomic number $\mathrm{Z}$ the critical angle below which total external reflection occurs is $\varphi_{\max }$ $(\mathrm{rad}) \approx 0.08 / E_{\gamma}(\mathrm{keV})$. For angles below the critical one reflectivity is close to unity. For our case, rhodium (Rh) is an ideal reflecting material. The reflecting mirrors may be placed downstream of the spectrometer at a distance of e.g. $10 \mathrm{~m}$ from the detector. Hard $\gamma$-quanta will pass through the reflector and only quanta with energies less than $20 \mathrm{keV}$ will be deflected from the main SR stream toward $\mathrm{x}$-values larger than $\pm 3 \mathrm{~cm}$. Application of such mirrors permits to solve several problems: selection of soft radiation photons with energies between 1-20 keV from hard $\gamma$-quanta, strong suppression of radiation doses within the detectors, and provision of high conversion efficiency of the photons to photoelectrons in the detector material.

\section{GEANT SIMULATION OF SYNCHRO- TRON RADIATION}

Synchrotron radiation light produced in the energy spectrometer magnets is simulated by means of GEANT [4]. GEANT simulation includes effects related to the energy spread of the electrons within a bunch, the bunch size, the magnetic fringe fields, and possible SR interactions within the thin steel vacuum chamber wall.

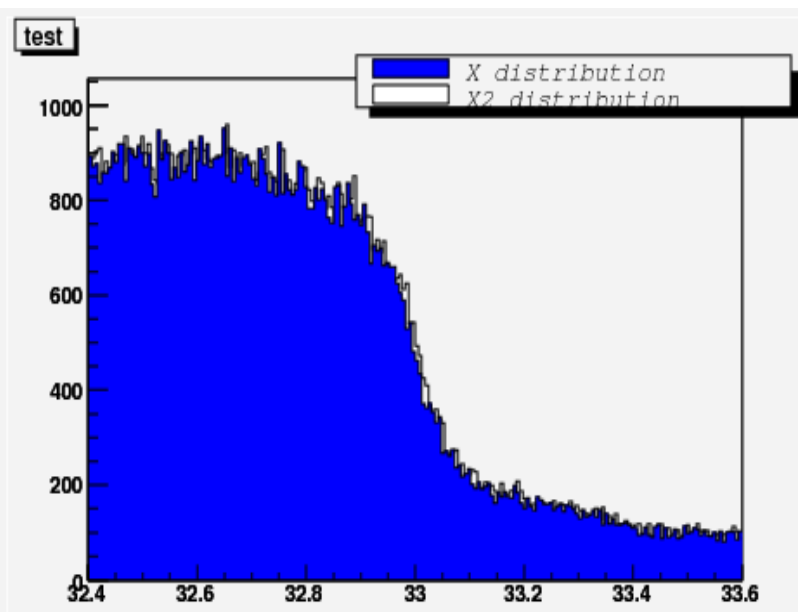

Figure 2: SR counting rate versus horizontal coordinate at electron energies of $250 \mathrm{GeV}$ and $250.05 \mathrm{GeV}$ (blue histogram) close to left SR fan horizontal edge.

A beam energy variation of $50 \mathrm{MeV}$ from the nominal energy of $250 \mathrm{GeV}$ (corresponding to a relative energy uncertainty of $\Delta \mathrm{E} / \mathrm{E} \approx 2 \cdot 10^{-4}$ ) produces the displacement of the right edge position of $5 \mu \mathrm{m}$, while the shift of the left edge position is 7-8 $\mu \mathrm{m}$ (Fig.2), so the total width shrinks by $12 \mu \mathrm{m}$. If we assume that the simulated resolution will be conserved for a beam energy variation of $10 \mathrm{MeV}$, the sensitivity of the method will be $2.5 \mu \mathrm{m} / 10 \mathrm{MeV}$. Such sensitivity permits to reach a beam energy resolution of $\Delta \mathrm{E} / \mathrm{E} \approx 5 \cdot 10^{-5}$ provided the endpoints of the SR fan can be determined with $2 \mu \mathrm{m}$ precision.

If the detectors are positioned within Roman Pots separated by a thin foil from the beam vacuum, photon interactions do not alter the sharpness of the SR fan edges 
strongly. The edges become somewhat less sharp, but they are still good recognizable and shifts of edge positions were not observed.

\section{NOVAL DETECTORS WITH SUB- MICRON SPATIAL RESOLUTION}

Properties of the SR light arising from the passage of electrons through the ILC magnetic spectrometer demand that possible detectors should have a position resolution of 2-3 $\mu \mathrm{m}$ for two detectors with a sensitive area of some $3 \mathrm{~mm}$ in horizontal direction. The detectors can be an assembly of many individual channels or layers $2 \mu \mathrm{m}$ thick interleaved with dielectric of $0.03 \mu \mathrm{m}$.

Technology for production of such multilayer coatings is well developed and the structure needed is perfect from the point of view of crystallography. Methods of electronbeam sputtering and laser-induced evaporation were widely used with layer thickness precisely checked by a reflectometer. The active material of the detector should be a semiconducting medium. The choice of the material with the required conductivity should be subject to the following condition: the relaxation time of the volume charge in the material should not be smaller than $30 \%$ of the peaking time of the charge-sensitive amplifier, which in turn should not be smaller than about 10 ns and larger than the ILC bunch-to-bunch spacing time of about 300 ns. Out of all abundant materials, $S i$ is the best choice meeting the above condition [5].

The number of $\gamma$-quanta with energies below $20 \mathrm{keV}$ entering $1 \mathrm{~mm}$ active detector area was estimated as $\mathrm{N}_{\gamma} \approx$ $5 \cdot 10^{8}$, see above. If we assume that a photoelectron spends about $10 \mathrm{eV}$ for an ionization event in $\mathrm{Si}$, and $30 \%$ of the total photon energy is transformed to photoelectrons, we expect on average 150 electrons. Eventually, about $7 \cdot 10^{10}$ photoelectrons are produced within $1 \mathrm{~mm}$ active detector area, so that each $2 \mu \mathrm{m}$ detector layer contains about $1.5 \cdot 10^{8}$ secondary electrons. With an amplifier conversion factor of $50 \mathrm{mV} / \mathrm{pC}$ these electrons produce output signal amplitude of $1.4 \mathrm{~V}$. With the (realistic) assumption that the photon energy distribution in each layer is close to a constant, any charge measurement of the photoelectrons in each layer provides correctly the position distribution of the soft photons registered.

The structure of the proposed detector is schematically shown in Fig.3. Its sensitive region of $2 \mathrm{~mm}$ contains about $1000 \mathrm{Si}$ layers, each $2 \mu \mathrm{m}$ thick. The layers are separated by dielectric $\mathrm{SiO}$ with a $0.03 \mu \mathrm{m}$ thickness.

To read out the information, each layer of the assembly is surrounded with a gold contact $5 \mu \mathrm{m}$ wide and $0.9 \mu \mathrm{m}$ thick connected to the charge-sensitive amplifier. For charge collection, each layer is connected to the plus terminal of the external power supply. The detecting and read-out electronics may be arranged in a simple and compact scheme as used e.g. for the readout of he cathode strips of the muon-chambers at the ATLAS facility [6]. Together with $\mathrm{Si}$ detector a plane-parallel avalanche detector with gas amplification of $10-50$ and a linear resolution of 2-3 $\mu \mathrm{m}$ is discussed also. Schematically, the avalanche detector is a flat capacitor filled with xenon at a pressure of $100 \mathrm{~atm}$. The anode plane of the detector comprises Ni layers of $1 \mu \mathrm{m}$ separated from each other by $2 \mu \mathrm{m} \mathrm{SiO}$ dielectric. To eliminate edge effects, the sensitive elements are placed inside a plastic frame of $10 \times 10 \mathrm{~mm}^{2}$ covered by Al. The $10 \mathrm{~mm}$ by $10 \mathrm{~mm}$ entrance window of the detector is made of $1 \mathrm{~mm}$ beryllium foil. High voltage is applied to the beryllium cathode foil.

The distance between the anode and the cathode of the detector may less than $1 \mathrm{~mm}$ at a pressure of $100 \mathrm{~atm}$. For gas amplification of about 10 the transverse dimension of the avalanche will be within the $1 \mu \mathrm{m}$ mean free path of the photoelectrons.

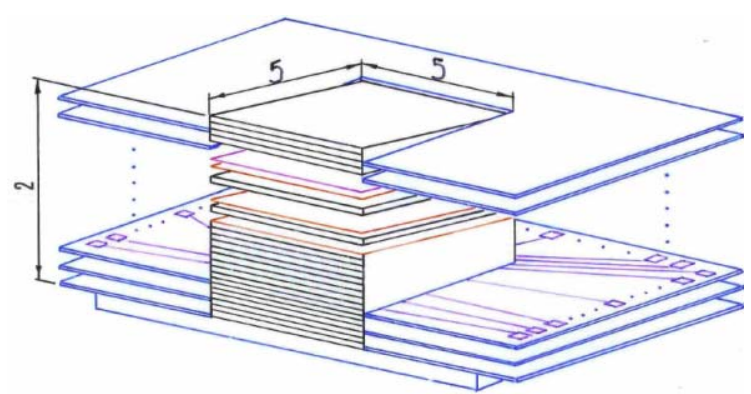

Figure 3: Semiconductor SR detector scheme with sub$\mu \mathrm{m}$ spatial resolution.

\section{CONCLUSION}

Precise position measurements of both horizontal edges of the SR fan permits to monitor the ILC beam energy with a precision of better than $\Delta E / E \cong 10^{-4}$.

Sensitivity estimates of the method from GEANT simulations result to $12 \mu \mathrm{m} / 50 \mathrm{MeV}$ for beam energies of $250 \mathrm{GeV}$. If this sensitivity is scaled to SR fan endpoint measurements with $2 \mu \mathrm{m}$ accuracy, beam energy measurements with a resolutions of $\Delta \mathrm{E} / \mathrm{E} \approx 5 \cdot 10^{-5}$ seem to be achievable.

Novel high-position resolution detectors for low energy gamma registration within large radiation background are proposed. Detector production however seems rather challenging and may be carried out by methods developed in microelectronic industry.

\section{REFERENCES}

[1] V. Duginov et al DESY Report, LC-DET-2004-031.

[2] J. Kent, M. King, C. Von Zanthier et al. Precision measurements of the SLC beam energy, PAC, Chicago, 1989.

[3] M. Levi, F. Rouse Precision Synchrotron radiation detectors, PAC, Chicago, 1989.

[4] GEANT4-Collaboration: e.g. K. Amako et al., Nucl. Instr. Meth. A453 (2000) 455.

[5] G. Dearnaley, D.C. Northrop Semiconductor counters for nuclear radiations, London, 1964.

[6] ATLAS Muon Spectrometer, Technical design report, June, 1977. 